\title{
Reversion From Mild Cognitive Impairment To Normal Cognition: False-Positive Error Or True Restoration Thanks To Cognitive Control Ability?
}

This article was published in the following Dove Press journal: Neuropsychiatric Disease and Treatment

\author{
Ji-Yeon Chung ${ }^{1,2, *}$ \\ Hyung-Jun Yoon (D) ${ }^{3, *}$ \\ Hoowon Kim (D) ${ }^{1,2}$ \\ Kyu Yeong Choi \\ Jang Jae Lee' \\ Kun Ho Lee (iD) ${ }^{1,4,5}$ \\ Eun Hyun Seo 1,6
}

'National Research Center for Dementia, Chosun University, Gwangju, Korea;

${ }^{2}$ Department of Neurology, College of Medicine, Chosun University/Chosun University Hospital, Gwangju, Korea; ${ }^{3}$ Department of Neuropsychiatry, College of Medicine, Chosun University/ Chosun University Hospital, Gwangju, Korea; ${ }^{4}$ Department of Biomedical Science, College of Natural Sciences, Chosun University, Gwangju, Korea; ${ }^{5}$ Department of Neural Development and Disease, Korea Brain Research Institute, Daegu, Korea; ${ }^{6}$ Department of Premedical Science, College of Medicine, Chosun University, Gwangju, Korea

*These authors contributed equally to this work

Correspondence: Eun Hyun Seo Premedical Science, College of Medicine, Chosun University, 309 Pilmundaero Dong-gu, Gwangju 61452, South Korea Tel +82 62-230-7664

Fax +82-62-229-1029

Email ehseo@chosun.ac.kr
Purpose: Relatively little attention has been paid to the meaning of reversion from mild cognitive impairment (MCI) to cognitively normal $(\mathrm{CN})$, compared to MCI progression studies. The purpose of the study was to investigate the characteristics contributing to reversion from $\mathrm{MCI}$ to $\mathrm{CN}$ and to identify the associated factors with such reversion.

Patients and methods: We retrospectively identified 200 individuals who initially diagnosed as MCI and completed the second visit from the National Research Center for Dementia (NRCD) registry in Korea. Participants underwent comprehensive clinical and neuropsychological assessments. Factors associated with reversion were examined by a independentsamples t-test, $\chi^{2}$ test, and logistic regression. Longitudinal change was examined by a repeated measures analysis of variance (rANOVA).

Results: Based on the second assessment, 78 (39\%) individuals were found to have reverted to $\mathrm{CN}$ (rMCI) and 118 (59\%) remained with MCI (sMCI). Four (2\%) progressed to Alzheimer's disease dementia and they were excluded from further analysis. Over a wide range of socio-demographic, clinical, and neuropsychological variables, group difference was significant only in neuropsychological tests of cognitive control. Both groups showed improvement in several neuropsychological tests, implying a practice effect, but the rMCI group showed greater improvement.

Conclusion: Reversion from MCI to $\mathrm{CN}$ might not be a false-positive error but a true recovery from cognitive impairment. Our results suggest that cognitive control ability may be a characteristic favorable for the restoration of cognitive function. Therefore, assessment of cognitive control might facilitate the development of appropriate interventions for MCI as well as prognosis evaluation.

Keywords: mild cognitive impairment, reversion, cognitive control, Alzheimer disease

\section{Introduction}

Mild cognitive impairment (MCI) is an intermediate state between normal aging and dementia. ${ }^{1}$ However, the natural progression of MCI is not always linear. ${ }^{2}$ For example, previous MCI conversion studies reported that considerable numbers of MCI cases can either be reversible ${ }^{3-7}$ or remain stable over a long period of time. ${ }^{8,9}$ Some important reasons for this inconsistent progression might be that MCI is a clinical entity describing early cognitive problems not serious enough to meet the criteria for dementia, and therefore it has various etiologies. MCI is heterogeneous in its neuropsychological profile ${ }^{10}$ and biomarker positivity. ${ }^{11}$ The issue of reversible cases of MCI has continuously emerged since its concept was introduced. ${ }^{1,12,13}$ Over 
the past decades, a number of studies have reported that cognitive impairment present at an initial assessment reverted to normal performance by the following assessment. $^{3,4,6,7,14-16}$ The incidence of reversion from MCI to $\mathrm{CN}$ varies from $8 \%$ to $59.3 \%,{ }^{17}$ according to the study setting (ie, community-based vs clinic-based); clinical characteristics of MCI; or the length of follow-up time.

Aside from the various reversion rates, understanding the meaning of reversion from MCI to $\mathrm{CN}$ is important for both research and clinical practice. There are controversies about possible reasons for such reversion. One possible reason is that true recovery from cognitive impairment has, in fact, occurred. ${ }^{3,5-7,16}$ If so, identifying the underlying mechanism or predictive factor for the cognitive recovery is crucial because it would provide prognostic values for dementia-related cognitive decline, as well as help with developing an intervention program. Secondly, the reversion could simply be false-positive errors at the initial diagnosis of $\mathrm{MCI},{ }^{14,15}$ which lead to modification of MCI criteria. ${ }^{18-20}$ As Brooks et $\mathrm{al}^{14}$ pointed out, the risk of over-interpretation of a low neuropsychological performance from one isolated assessment is especially high in older adults with low intelligence. Third, some etiologies causing MCI are more likely to exhibit cognitive fluctuations. Some causes of MCI other than Alzheimer's disease (AD) could result in recovery or improvement by the second evaluation. Some of these other causes may include neuropsychiatric symptoms ${ }^{21}$ or vascular pathology with a subsequent repair mechanism. ${ }^{22}$ Lastly, reversion to $\mathrm{CN}$ could occur from the practice effects at the second assessment. Repetitive exposure to neuropsychological test can result in a practice effect in $\mathrm{MCI}^{23-25}$ as well as normal aging. ${ }^{26,27}$ However, the literature on the meaning of reversion from $\mathrm{MCI}$ to $\mathrm{CN}$ remains inconclusive. Relatively little attention has been paid to elucidate the characteristics contributing to reversion from MCI to $\mathrm{CN}$, compared to MCI progression studies.

Furthermore, it is imperative to find positive prognostic factors in MCI. It is unclear whether specific cognitive characteristics or clinical factors are useful for predicting reversion from $\mathrm{MCI}$ to $\mathrm{CN}$ or not. A limited number of studies reported clinical factors associated with reversion to $\mathrm{CN}$. However, the results were inconsistent across studies. For example, Pandya et al reported MCI with younger age and no apolipoprotein E (APOE) were more likely to revert, ${ }^{3}$ whereas Sachdev et al reported no age or APOE effect on reversion. ${ }^{6}$ Sachdev et al reported MCI with more educated were likely to revert, ${ }^{6}$ whereas
Koepsell et al reported no education effect on reversion. ${ }^{7}$ Heterogeneity of the MCI group in previous studies made it difficult to systematically focus on underlying factors for reversion. There is a need for the full characterization of reversion from $\mathrm{MCI}$ to $\mathrm{CN}$ in a more homogenous $\mathrm{MCI}$ group. Therefore, the purpose of the study was to investigate the characteristics contributing to reversion from MCI to $\mathrm{CN}$ and to identify clinical and neuropsychological predictive factors associated with such reversion.

\section{Materials And Methods}

\section{Participants}

We included individuals with MCI recruited from a pool of individuals registered at the National Research Center for Dementia (NRCD) in Gwangju, Korea. We retrospectively selected data only if they had completed second follow-up evaluation. The NRCD database was described previously. ${ }^{28,29}$ It consists of elderly people aged 60 and over who volunteered to participate. Requests for volunteers were made through local newspapers and posters placed on a bulletin board at public health centers and senior centers. All participants were community-dwelling and fully informed regarding study participation. Evaluation procedure consists of three stages: 1) Initial brief interview including demographic information, medical history, the Mini-Mental State Examination (MMSE), and selfreport questionnaires (Day 1). 2) Detailed evaluation including comprehensive neuropsychological assessments and MRI scan (Day 2). 3) Diagnostic interview including clinical dementia rating (CDR) and diagnosis (Day 3). Initial brief interview and neuropsychological assessments were administered by trained psychologists. Diagnostic interview was performed by dementia experts (four neurologists and one neuropsychiatrists). The final clinical diagnosis was made after reviewing all the available information in the consensus case conferences. MCI was diagnosed according to the following consensus criteria: ${ }^{30}$ a) the person is neither normal nor demented; $b$ ) there is evidence of cognitive deterioration shown by a subjective report of decline by the self and/or an informant as well as objective cognitive deficits; and c) activities of daily living are preserved, and complex instrumental functions are either intact or minimally impaired. In terms of the criteria for (b), objective cognitive deficits were identified by the neuropsychological performance z-scores based on normative data. That is, objective cognitive deficit was defined based on if the score was 1.5 standard deviations (SD) below normative means on at least one test among the neuropsychological assessment battery. All had an overall CDR of 0.5 . In addition, to obtain as much of a 
homogeneous MCI group as possible, careful exclusion criteria for all MCI participants were applied as follows: 1) evidence of focal brain lesions on MRI, including lacunas and white matter hyperintensity lesions of grade 2 or more, according to the Fazekas scale $;^{31}$ 2) any significant neurologic, medical, or neuropsychiatric disorders (eg, depression or anxiety disorders) that could affect mental function in either past or present medical history; and 3) current use of psychoactive medications. It was 743 individuals who were diagnosed as MCI at the first assessment. Among them, we excluded 216 individuals in the current study because they met exclusion criteria. The second neuropsychological assessment was performed for 243 individuals with MCI but 43 did not complete diagnostic interview (Day 3). Finally, we identified 200 participants who were diagnosed with MCI at baseline from March 2014 to July 2016 and reassessed them at a subsequent visit about 1.5 years later (second visit duration was from June 2015 to November 2017). Four clinical MCI subtypes were classified as either amnestic MCI (aMCI)-single domain, aMCImultiple domain, nonamnestic MCI (naMCI)-single domain, and naMCI-multiple domain, based on the impaired cognitive domain participants had.

\section{Baseline Clinical And Neuropsychological Assessment}

All participants were examined with a clinical interview, which included an assessment of CDR. ${ }^{32,33}$ Instrumental activities of daily living (IADL) ${ }^{34}$ were also assessed. Information on major socio-demographic variables, medical history, family history for dementia and stroke, and body mass index (BMI) were collected. For BMI, 18.522.9 was considered to be normal weight, 23-24.9 was considered to be at risk, and $\geq 25$ as obese. ${ }^{35}$ The Dementia Screening Questionnaire (DSQ), ${ }^{36}$ a self-report format of a cognitive screening tool, was also administered. In addition, the Subjective Memory Complaints (SMC) ${ }^{37}$ and the Geriatric Depression Scale (GDS) ${ }^{38}$ were also administered in a self-report format.

A comprehensive neuropsychological assessment was performed. The attention domain was assessed using a digit span forward (DSF) and digit span backward (DSB). The language domain was assessed using a shortened version of the Boston Naming Test (BNT; 15 item version, Form A). The visuospatial domain was assessed using the copying test from the Rey Complex Figure Test (RCFT copy). The memory domain was assessed by six measures, including the Seoul Verbal Learning Test (SVLT) immediate recall (SVLT_imm), 20-min delayed recall (SVLT_delayed), yes-no recognition (SVLT_rec), RCFT immediate recall (RCFT_imm), 20-min delayed recall (RCFT_delayed), and yes-no recognition (RCFT_rec). The executive function domain was assessed using fluency test for animal, a phonemic fluency test (total score for " $\neg$ "/g/, “人”/s/, and "O"/y/), the Stroop test (color naming in colorword incongruent conditions), and the Trail Making Tests (TMT) A and B. Global cognition was assessed using the MMSE. Neuropsychological performances were transformed to z-scores based on age-, education-, and gender-specific normative data. $^{39}$

\section{Determination Of APOE $\varepsilon 4$ Genotype}

The procedure for determination of the apolipoprotein (APOE) genotype was previously described. ${ }^{40}$ Briefly, genomic DNA was extracted from buffy coats isolated from whole blood and the APOE genotype was determined by the single-nucleotide polymorphisms of rs429358 and rs7412. The APOE $\varepsilon 4$ positive (APOE $\varepsilon^{+}$) genotype was assigned if at least one $\varepsilon 4$ allele was present.

\section{Follow-Up Assessment}

Each participant underwent the same procedure as they did in the baseline evaluation. In addition to MCI diagnosis, reversion to $\mathrm{CN}$ was defined if all of the neuropsychological performance scores were within a normal range and the CDR score was 0 at the follow-up assessment. In the case of progression, on the other hand, dementia was diagnosed according to the Diagnostic and Statistical Manual of Mental Disorders (DSM-IV) criteria for dementia and the National Institute of Neurological and Communication Disorders and Stroke/Alzheimer's Disease and Related Disorders Association (NINCDS-ADRDA) criteria for probable AD. ${ }^{41,42}$

\section{Statistical Analysis}

The MCI group was divided into two subgroups according to diagnosis at the time of the second evaluation: those who reverted to $\mathrm{CN}$ (rMCI) and those who stayed at MCI (sMCI). Demographic characteristics and comprehensive clinical information were compared between groups using a independent-samples t-test and $\chi^{2}$ test for continuous and categorical variables, respectively. For neuropsychological performance, z-scores were compared between groups using a independent-samples t-test. Group comparisons for number of impaired neuropsychological tests were performed using Mann-Whitney U-test because they were non-normally distributed. Multivariable logistic 
regression analysis with forward stepwise selection was performed to examine the role of neuropsychological test scores at baseline as predictors of reversion from MCI to $\mathrm{CN}$. In addition, a two-way repeated measure analysis of variance (rANOVA) was conducted to compare cognitive changes between rMCI and SMCI in terms of longitudinal perspective. These analyses were performed using SPSS version 25.0 for Windows (SPSS Inc., Chicago, IL) Pvalues less than 0.05 were considered significant. To control for type I errors, false discovery rate (FDR) correction for multiple comparisons was applied using BenjaminiHochberg step-up procedure, the mean FDR $\alpha .^{43}$ For effect sizes, Cohen's d, Phi, $\eta^{2}$, and $\eta_{p}^{2}$ were calculated for t-test, $\chi^{2}$ test, Mann-Whitney U-test, and rANOVA, respectively.

\section{Ethics Statement}

The institutional review board of Chosun University Hospital approved the present study. Written informed consent was obtained from each participant or legal guardians. This study was conducted in accordance with the Declaration of Helsinki.

\section{Results}

\section{Participant Characteristics}

The mean period of follow-up was $18.16 \pm 6.59$ months for the entire group. At the second visit, 118 (59\%) were diagnosed with $\mathrm{MCI}$ again and they were classified as sMCI. Seventy-eight (39\%) reverted to $\mathrm{CN}$ and they were classified as rMCI. Lastly, $4(2 \%)$ had progressed to AD dementia and they were excluded from further analysis because the focus of current study is reversion rather than progression. Participants from the rMCI group were less educated than those from sMCI group $(\mathrm{p}=$ 0.034). There were no group differences in age, gender, follow-up duration, economic status, family history of dementia and stroke, BMI, former job complexity, and APOE $\varepsilon 4$ positivity (see Table 1).

Table I Demographic Characteristics At Baseline In Mild Cognitive Impairment Subgroups

\begin{tabular}{|c|c|c|c|c|c|c|}
\hline & $\mathrm{rMCl}(\mathrm{n}=78)$ & $\operatorname{sMCl}(n=1 \mid 8)$ & $\mathbf{t}$ & $\chi^{2}$ & $P$ value & d/phi \\
\hline Age, y & $73.42 \pm 4.75$ & $74.00 \pm 4.83$ & -0.824 & & 0.411 & 0.120 \\
\hline Education, y & $10.12 \pm 3.66$ & I I.44 \pm 4.58 & -2.141 & & 0.034 & 0.312 \\
\hline Female, n (\%) & $44(56.4)$ & $50(42.4)$ & & 3.707 & 0.054 & 0.138 \\
\hline FU & $18.73 \pm 6.45$ & $17.79 \pm 6.67$ & 0.982 & & 0.327 & 0.143 \\
\hline APOE $\varepsilon^{+}, \mathrm{n}(\%)$ & $18(23.4)$ & $28(24.3)$ & & 0.024 & 0.877 & 0.011 \\
\hline $\begin{array}{l}\text { Economic status, n (\%) } \\
\text { Low } \\
\text { Moderate } \\
\text { High }\end{array}$ & $\begin{array}{l}31(39.7) \\
35(44.9) \\
12(15.4)\end{array}$ & $\begin{array}{l}35(29.7) \\
51(43.2) \\
32(27.1)\end{array}$ & & 4.327 & 0.115 & 0.149 \\
\hline $\begin{array}{l}\text { Family history, n (\%) } \\
\text { Dementia } \\
\text { Stroke }\end{array}$ & $\begin{array}{l}14(17.9) \\
18(23.1)\end{array}$ & $\begin{array}{l}21(17.8) \\
16(13.6)\end{array}$ & & $\begin{array}{l}0.001 \\
2.967\end{array}$ & $\begin{array}{l}0.978 \\
0.085\end{array}$ & $\begin{array}{l}0.002 \\
0.123\end{array}$ \\
\hline $\begin{array}{c}\text { BMI, n (\%) } \\
\text { Normal } \\
\text { At risk } \\
\text { Obese }\end{array}$ & $\begin{array}{l}18(23.1) \\
19(24.4) \\
41(52.6)\end{array}$ & $\begin{array}{l}33(28.7) \\
32(27.8) \\
50(43.5)\end{array}$ & & 1.580 & 0.454 & 0.090 \\
\hline $\begin{array}{l}\text { Job complexity, n (\%) } \\
\text { Unemployed } \\
\text { Low } \\
\text { Moderate } \\
\text { High }\end{array}$ & $\begin{array}{l}28(35.9) \\
14(17.9) \\
19(24.4) \\
17(21.8)\end{array}$ & $\begin{array}{l}27(22.9) \\
22(18.6) \\
23(19.5) \\
46(39.0)\end{array}$ & & 7.683 & 0.053 & 0.198 \\
\hline
\end{tabular}

Notes: Data are presented as mean \pm standard deviation, unless specified otherwise. The bolded line indicates significant group difference.

Abbreviations: $\mathrm{rMCl}$, mild cognitive impairment group who reverted to cognitive normal state at follow-up; sMCl, mild cognitive impairment group who stayed as $\mathrm{MCl}$ at follow-up; $d$, Cohen's $d$ value measuring effect size for t-test; phi, phi value measuring effect size for $\chi^{2}$ test; FU, mean follow-up time in month; APOE $\varepsilon^{+}$, apolipoprotein $\varepsilon 4$ carrier; BMI, body mass index. 


\section{Clinical And Neuropsychological Characteristics At Baseline}

Clinical and neuropsychological characteristics at baseline evaluation are shown in Table 2. SMC, IADL, DSQ, and
GDS were not significantly different between the rMCI and sMCI groups. Regarding four clinical subtypes, the aMCI-multiple domain type was the most frequent, and the naMCI-multiple domain type was the least frequent.

Table 2 Clinical And Neuropsychological Characteristics At Baseline In Mild Cognitive Impairment Subgroups

\begin{tabular}{|c|c|c|c|c|c|c|}
\hline & $\mathrm{rMCl}(\mathrm{n}=78)$ & $\operatorname{sMCl}(n=118)$ & $\mathbf{t}$ & $\chi^{2}$ & $P$ value & d/phi \\
\hline SMC & $4.46 \pm 3.24$ & $3.67 \pm 2.94$ & 1.672 & & 0.096 & 0.244 \\
\hline IADL & $0.42 \pm 0.76$ & $0.62 \pm 1.44$ & -1.032 & & 0.303 & 0.151 \\
\hline DSQ & $4.74 \pm 2.93$ & $4.64 \pm 2.61$ & 0.270 & & 0.788 & 0.039 \\
\hline GDS & $10.63 \pm 6.49$ & $8.59 \pm 6.33$ & 2.180 & & 0.030 & 0.318 \\
\hline MMSE & $-0.72 \pm 1.25$ & $-0.77 \pm 1.16$ & 0.297 & & 0.767 & 0.043 \\
\hline $\begin{array}{l}\mathrm{MCl} \text { subtypes, } \mathrm{n}(\%) \\
\text { aMCl_single } \\
\text { aMCl_multiple } \\
\text { naMCl_single } \\
\text { naMCl_multiple }\end{array}$ & $\begin{array}{l}30(38.5) \\
34(43.6) \\
9(11.5) \\
5(6.4)\end{array}$ & $\begin{array}{l}26(22.0) \\
62(52.5) \\
17(14.4) \\
13(11.0)\end{array}$ & & 6.580 & 0.087 & 0.183 \\
\hline $\begin{array}{l}\text { Attention } \\
\text { DSF } \\
\text { DSB }\end{array}$ & $\begin{array}{l}-0.19 \pm 1.13 \\
-0.38 \pm 0.76\end{array}$ & $\begin{array}{l}-0.10 \pm 1.13 \\
-0.46 \pm 1.01\end{array}$ & $\begin{array}{l}-0.549 \\
0.559\end{array}$ & & $\begin{array}{l}0.583 \\
0.577\end{array}$ & $\begin{array}{l}0.080 \\
0.082\end{array}$ \\
\hline $\begin{array}{l}\text { Language } \\
\text { BNT }\end{array}$ & $0.10 \pm 1.02$ & $-0.04 \pm \mathrm{I} . \mathrm{II}$ & 0.883 & & 0.379 & 0.129 \\
\hline $\begin{array}{l}\text { Visuospatial functions } \\
\text { RCFT copy }\end{array}$ & $0.10 \pm 0.91$ & $-0.27 \pm 1.05$ & 2.562 & & 0.011 & 0.374 \\
\hline $\begin{array}{l}\text { Memory } \\
\text { SVLT_imm } \\
\text { SVLT_delayed } \\
\text { SVLT_rec } \\
\text { RCFT_imm } \\
\text { RCFT_delayed } \\
\text { RCFT_rec }\end{array}$ & $\begin{array}{l}-0.62 \pm 0.98 \\
-0.74 \pm 1.00 \\
-0.52 \pm 1.20 \\
-0.41 \pm 1.10 \\
-0.49 \pm 0.97 \\
-0.33 \pm 0.93\end{array}$ & $\begin{array}{l}-0.89 \pm 0.77 \\
-0.96 \pm 0.81 \\
-0.70 \pm 1.10 \\
-0.69 \pm 0.91 \\
-0.65 \pm 0.88 \\
-06 \pm 0.94\end{array}$ & $\begin{array}{l}2.156 \\
1.708 \\
1.065 \\
1.927 \\
1.144 \\
0.234\end{array}$ & & $\begin{array}{l}0.032 \\
0.089 \\
0.288 \\
0.055 \\
0.254 \\
0.815\end{array}$ & $\begin{array}{l}0.315 \\
0.249 \\
0.155 \\
0.281 \\
0.167 \\
0.034\end{array}$ \\
\hline $\begin{array}{l}\text { Executive functions } \\
\text { Fluency_A } \\
\text { Fluency_P } \\
\text { Stroop_W } \\
\text { Stroop_C } \\
\text { TMT_A } \\
\text { TMT_B }\end{array}$ & $\begin{array}{l}-0.40 \pm 0.97 \\
-0.04 \pm 0.93 \\
0.09 \pm 1.14 \\
-0.22 \pm 1.02 \\
0.02 \pm 0.87 \\
-0.01 \pm 1.02\end{array}$ & $\begin{array}{l}-0.43 \pm 0.80 \\
-0.48 \pm 0.92 \\
-0.21 \pm 1.13 \\
-0.55 \pm 1.19 \\
-0.42 \pm 1.17 \\
-0.40 \pm 1.03\end{array}$ & $\begin{array}{l}0.234 \\
3.248 \\
1.822 \\
1.993 \\
2.796 \\
2.490\end{array}$ & & $\begin{array}{l}0.815 \\
\mathbf{0 . 0 0 1} \\
0.070 \\
0.048 \\
\mathbf{0 . 0 0 6} \\
\mathbf{0 . 0 1 4}\end{array}$ & $\begin{array}{l}0.034 \\
0.474 \\
0.266 \\
0.291 \\
0.408 \\
0.363\end{array}$ \\
\hline
\end{tabular}

Notes: Data are presented as mean \pm standard deviation, unless specified otherwise. Neuropsychological test scores are group mean z-scores based on age-, education-, and gender-specific normative information \pm standard deviation. The bolded lines indicate significant group difference at $p<0.05$ after false discovery rate correction for multiple comparison.

Abbreviations: $\mathrm{rMCl}$, mild cognitive impairment group who reverted to cognitive normal state at follow-up; sMCl, mild cognitive impairment group who stayed as $\mathrm{MCl}$ at follow-up; d, Cohen's d value measuring effect size for t-test; phi, phi value measuring effect size for $\chi^{2}$ test; aMCl, amnestic mild cognitive impairment; naMCl, nonamnestic mild cognitive impairment; SMC, subjective memory complaints; IADL, instrumental activities of daily living; DSQ, dementia screening questionnaire; GDS, geriatric depression scale; MMSE, mini mental status examination; DSF, digit span forward; DSB, digit span backward; BNT, Boston naming test (I5 items); RCFT copy, Rey complex figure test copy score; SVLT_imm, Seoul verbal learning test, immediate recall score; SVLT_delayed, Seoul verbal learning test delayed recall score; SVLT_rec, Seoul verbal learning test recognition score; RCFT_imm, Rey complex figure test immediate recall score; RCFT_delayed, Rey complex figure test delayed recall score; RCFT_rec, Rey complex figure test recognition score; Fluency_A, fluency score for animal; Fluency_P, fluency score for 3 Korean alphabets; Stroop_W, Stroop score for word reading; Stroop_C, Stroop score for color naming in color-word in incongruent condition; TMT, trail making test. 
The distribution of four MCI subtypes was not different between groups $\left(\chi_{\mathrm{df}=3}^{2}=6.58, \mathrm{p}=0.087\right)$. In terms of aMCI versus naMCI, $42.1 \%$ and $31.8 \%$ reverted to $\mathrm{CN}$, respectively, and the distribution was not different between groups $\left(\chi_{\mathrm{df}=1}^{2}=1.507, \mathrm{p}=0.220\right)$.

For neuropsychological tests, z-scores of RCFT copy, Fluency_P, TMT_A, and TMT_B were significantly higher in the rMCI group than those in the sMCI group. No neuropsychological z-scores resulted higher in the sMCI group than the rMCI group. On the other hand, no group difference was found in the mean number of impaired neuropsychological tests $(p=0.095)$. Mann-Whitney Utest also revealed no group difference in the number of impaired tests from memory $\left(p=0.509, \eta^{2}=0.002\right)$ executive function $\left(p=0.554, \eta^{2}=0.019\right)$, and other cognitive function ( $p=0.063, \eta^{2}=0.018$ ). In addition, the frequency of MCI with only one impaired test was not different between groups $\left(\chi_{\mathrm{df}=1}^{2}=1.89, \mathrm{p}=0.17\right)$.

\section{Associations Of Reversion From $\mathrm{MCl}$ To CN With The Neuropsychological Test Scores At Baseline}

Multivariable logistic regression analysis with forward stepwise selection was performed to examine the ability of neuropsychological test scores at baseline to predict reversions from $\mathrm{MCI}$ to $\mathrm{CN}$. The odds ratio (OR) listed in Table 3 refers to association with reversion to $\mathrm{CN}$, not with progression to dementia. As shown in Table 3, demographic variables were entered at the first step to control their influence on reversion. Then, neuropsychological test z-scores, which had shown significant group difference resulted from the t-tests, were entered at the second step. Fluency_P (OR $=1.653 ; 95 \% \mathrm{CI} ; 1.156-2.364 ; \mathrm{p}=0.006)$ and TMT_A (OR=1.603; 95\% CI; 1.091-2.355; $\mathrm{p}=0.015)$ remained as significant predictors for reversion to $\mathrm{CN}$.

\section{Reversion From $\mathrm{MCl}$ To $\mathrm{CN}$ And Longitudinal Cognitive Change}

Longitudinal cognitive changes in $\mathrm{rMCI}$ and sMCI were compared by a two-way rANOVA. It revealed a group of tests showing within- and between-group effects or interaction effects (Table 4 and Figure 1). The RCFT copy, Fluency_P, Stroop_C, and all memory scores except the RCFT_rec showed both within-group and between-group effects significantly. These scores significantly improved at follow-up assessment in both groups, but the rMCI group showed a significantly higher level of scores in the assessments overall. On the other hand, some tests showed either the betweengroup effect (BNT, Strooo_W, TMT_A, and TMT_B) or within-group effect (RCFT_rec) significantly. The scores of the BNT, Stroop_W, TMT_A, and TMT_B in the rMCI group were significantly higher than those in the sMCI group, but there were no longitudinal changes. The RCFT_rec score was improved at follow-up assessment, but it was not different between rMCI and sMCI group. Lastly, there were significant interaction effects in DSF, Fluency_A, and four memory scores. The rMCI group exhibited improvement in DSF and fluency_A scores whereas the sMCI group exhibited a slight decrease in the scores. Memory scores improved in both groups, but the rMCI group exhibited significantly greater improvement than the sMCI group (Figure 1).

\section{Discussion}

Understanding the characteristics of reversion from MCI to $\mathrm{CN}$ is important in both research and clinical settings. In this retrospective study, we analyzed a variety of factors in older adults with MCI who reverted and remained stable after a 1.5-year follow-up. Comparing baseline characteristics between rMCI and sMCI revealed that individuals with MCI with a relatively higher performance on RCFT

Table 3 Logistic Regression Analysis For Factors Associated With Reversion To Normal Cognition

\begin{tabular}{|c|c|c|c|c|c|c|c|c|c|c|}
\hline \multirow[t]{2}{*}{ Variables } & \multicolumn{5}{|c|}{ Step $I^{a}$} & \multicolumn{5}{|c|}{ Step $2^{b}$} \\
\hline & B & SE & Wald & $P$ value & OR $(95 \% \mathrm{Cl})$ & B & SE & Wald & P value & OR $(95 \% \mathrm{Cl})$ \\
\hline Gender & 0.179 & 0.354 & 0.257 & 0.612 & I.196 (0.598-2.395) & 0.566 & 0.394 & 2.070 & 0.150 & $1.762(0.814-3.812)$ \\
\hline Age & -0.010 & 0.034 & 0.090 & 0.764 & $0.990(0.925-1.059)$ & -0.008 & 0.036 & 0.052 & 0.819 & $0.992(0.924-1.065)$ \\
\hline Education & -0.102 & 0.046 & 4.910 & 0.027 & $0.903(0.825-0.988)$ & -0.092 & 0.049 & 3.468 & 0.063 & $0.912(0.828-1.005)$ \\
\hline Fluency_P & & & & & & 0.503 & 0.183 & 7.576 & 0.006 & $1.653(1.156-2.365)$ \\
\hline TMT_A & & & & & & 0.472 & 0.196 & 5.790 & 0.016 & $1.603(1.091-2.355)$ \\
\hline
\end{tabular}

Notes: ${ }^{a} \chi^{2}$ of model $=8.08, \mathrm{df}=3$, Nagelkerke $\mathrm{R}^{2}=0.06, \mathrm{p}=0.044 ;{ }^{\mathrm{b}} \chi^{2}$ of model $=25.62, \mathrm{df}=5$, Nagelkerke $\mathrm{R}^{2}=0.20, \mathrm{p}<0.00 \mathrm{I}$

Abbreviations: Fluency_P, fluency score for 3 Korean alphabets; TMT_A, trail making test part A. 
Table 4 Longitudinal Changes In Neuropsychological Test Scores Between Mild Cognitive Impairment Subgroups

\begin{tabular}{|c|c|c|c|c|c|c|}
\hline & \multicolumn{2}{|c|}{ Within Group } & \multicolumn{2}{|c|}{ Between Group } & \multicolumn{2}{|c|}{ Interaction } \\
\hline & $P$ value & $\eta_{p}^{2}$ & $P$ value & $\eta_{p}^{2}$ & $P$ value & $\eta_{p}^{2}$ \\
\hline \multicolumn{7}{|l|}{ Attention } \\
\hline DSF & 0.177 & 0.009 & 0.183 & 0.009 & 0.004 & 0.042 \\
\hline DSB & 0.845 & 0.000 & 0.183 & 0.009 & 0.306 & 0.005 \\
\hline \multicolumn{7}{|l|}{ Language } \\
\hline BNT & $0.74 I$ & 0.001 & 0.022 & 0.027 & 0.044 & 0.021 \\
\hline \multicolumn{7}{|l|}{ Visuospatial functions } \\
\hline RCFT copy & 0.001 & $0.05 I$ & 0.001 & 0.056 & 0.846 & 0.000 \\
\hline \multicolumn{7}{|l|}{ Memory } \\
\hline SVLT_imm & $<0.001$ & 0.262 & $<0.001$ & 0.062 & 0.021 & 0.027 \\
\hline SVLT_delayed & $<0.001$ & 0.272 & $<0.001$ & 0.083 & $<0.001$ & 0.084 \\
\hline SVLT_rec & $<0.001$ & 0.110 & 0.001 & 0.058 & 0.005 & 0.041 \\
\hline RCFT_imm & $<0.001$ & 0.062 & 0.005 & 0.041 & 0.135 & 0.012 \\
\hline RCFT_delayed & $<0.001$ & 0.119 & 0.017 & 0.030 & 0.020 & 0.028 \\
\hline RCFT_rec & 0.020 & 0.028 & 0.095 & 0.015 & 0.090 & 0.015 \\
\hline \multicolumn{7}{|l|}{ Executive functions } \\
\hline Fluency_A & 0.310 & 0.005 & 0.094 & 0.014 & 0.017 & 0.029 \\
\hline Fluency_P & 0.019 & 0.028 & $<0.001$ & 0.063 & 0.728 & 0.001 \\
\hline Stroop_W & 0.052 & 0.020 & 0.007 & 0.038 & 0.265 & 0.007 \\
\hline Stroop_C & $<0.001$ & 0.147 & 0.006 & 0.038 & 0.239 & 0.007 \\
\hline TMT_A & 0.179 & 0.010 & $<0.001$ & 0.066 & 0.577 & 0.002 \\
\hline TMT_B & 0.271 & 0.008 & $<0.001$ & 0.116 & 0.918 & 0.000 \\
\hline
\end{tabular}

Notes: The bolded values indicate significant group difference at $p<0.05$ after false discovery rate correction for multiple comparisons.

Abbreviations: $\eta_{p}^{2}$, partial eta-squared measuring effect size; DSF, digit span forward; DSB, digit span backward; BNT, Boston naming test (I5 items); RCFT copy, Rey complex figure test copy score; SVLT_imm, Seoul verbal learning test immediate recall score; SVLT_delayed, Seoul verbal learning test delayed recall score; SVLT_rec, Seoul verbal learning test recognition score; RCFT_imm, Rey complex figure test immediate recall score; RCFT_delayed, Rey complex figure test delayed recall score; RCFT_rec, Rey complex figure test recognition score; Fluency_A, fluency score for animal; Fluency_P, fluency score for 3 Korean alphabets; Stroop_W, Stroop score for word reading; Stroop_C, Stroop score for color naming in color-word in incongruent condition; TMT, trail making test.

copy, Fluency_P, TMT_A, and TMT_B were more likely to revert. Fluency_P and TMT_A remained significant in the final model for predicting reversion. Both the rMCI and sMCI groups showed improved performance in some cognitive tests, but the rMCI group showed much greater improvement at follow-up assessment.

Thirty-nine percent (78 out of 200 participants) reverted to normal cognition. Although the incidence rate of reversion varied depending on the subject source or follow-up period, our result is fairly consistent with the previous reports that used community-based recruitment. ${ }^{4,6,44}$ It is not surprising that a recent meta-analysis based on 25 studies reported that reversion rate was much higher in community-based studies (eg, 31\%) than clinic-based ones (eg, 14\%). ${ }^{45}$ Clinic-based samples consist of individuals who seek medical service for their cognitive problems; therefore, they are more likely have greater cognitive deficits than community-based samples. The source of subjects can influence the course of MCI. ${ }^{46,47}$
Our rMCI and sMCI groups were not different in terms of socio-demographic variables except education. For education, the rMCI group was less educated than the sMCI group ( $\mathrm{p}=0.034$ ) when using simple comparison, but the significance disappeared in the multivariable logistic regression model (Table 3), which shows a consistent pattern with a previous study. ${ }^{6}$ Although one study reported higher education in the reverted group, ${ }^{5}$ the sample size was small (6 reverters out of the 74 subjects). Therefore, the influence of education on reversion may be little or minor. There were no significant differences in clinical characteristics including APOE $\varepsilon 4$ positivity, subjectively reported memory and cognitive symptoms, subjective depression level, and daily functional status at baseline. These results indicate that the rMCI and sMCI groups in the current study had very similar backgrounds in general. Moreover, global cognitive level, distribution of clinical MCI subtypes and the number of z-scores that 

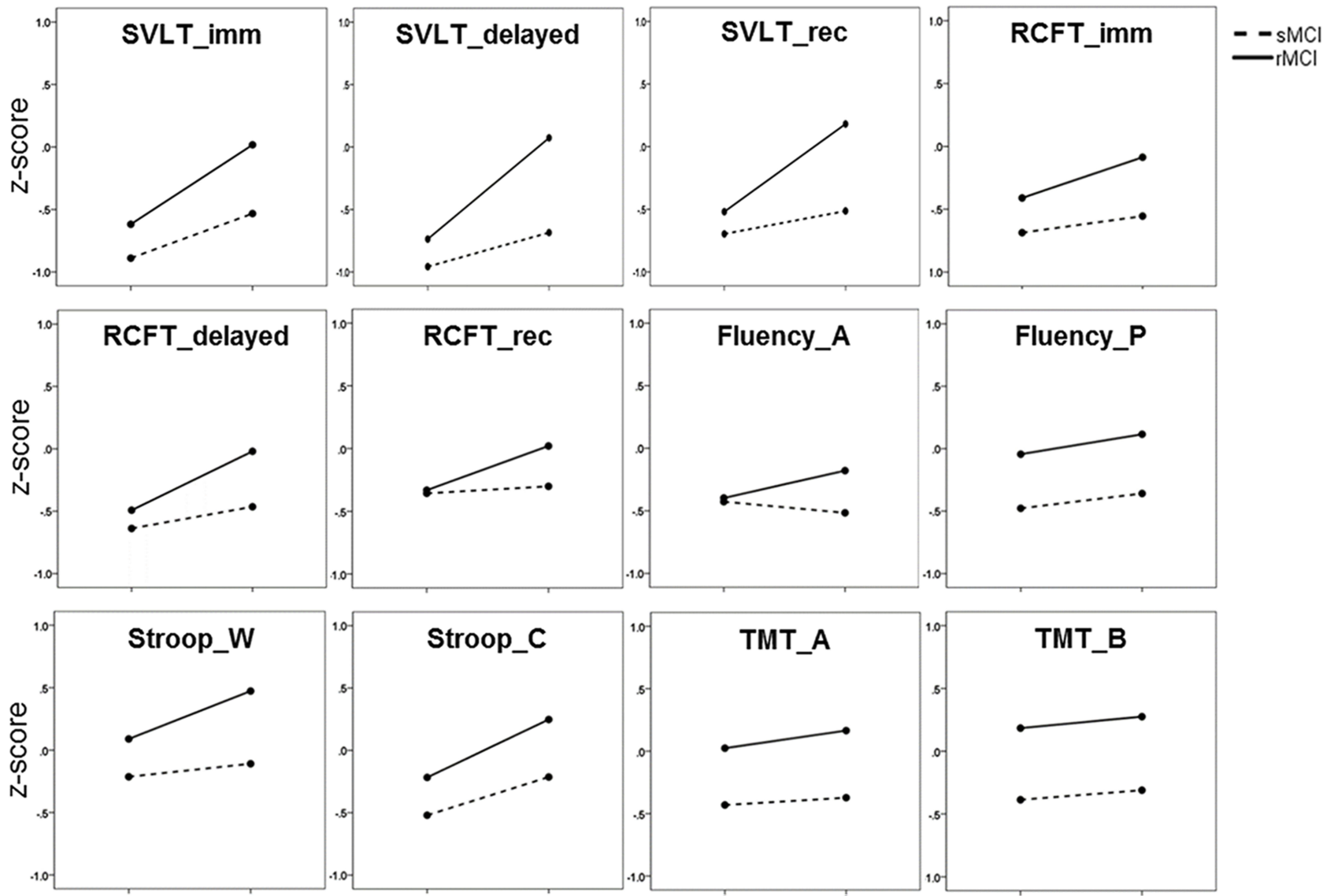

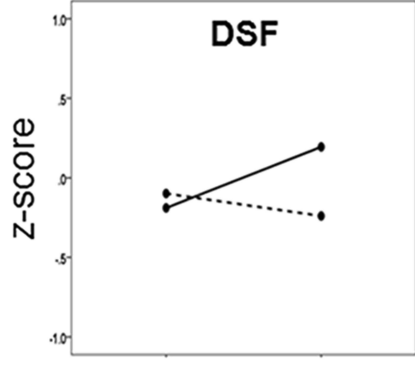

T1

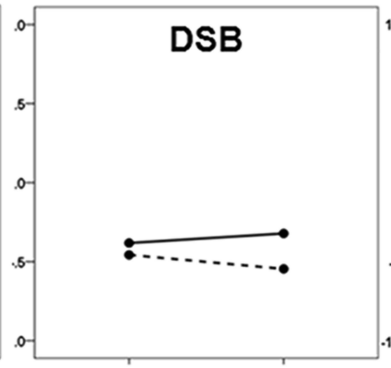

T1

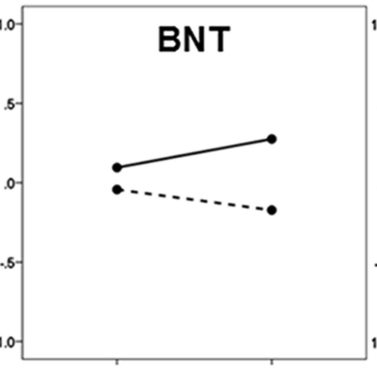

T1

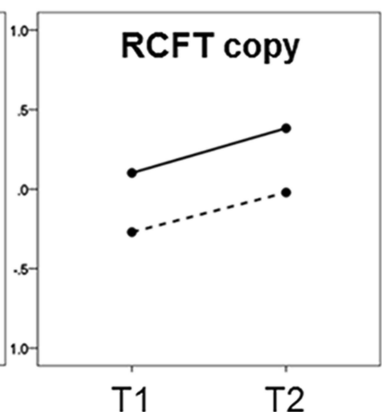

Figure I Changes in neuropsychological test scores in mild cognitive impairment subgroups.

Notes: Solid line indicates $\mathrm{rMCl}$; Dashed line indicates $\mathrm{sMCl}$.

Abbreviations: $\mathrm{rMCl}$, mild cognitive impairment group who reverted to cognitive normal state at follow-up; sMCl, mild cognitive impairment group who stayed as $\mathrm{MCl}$ at follow-up; TI, score at initial assessment; T2, score at the second assessment; SVLT_imm, Seoul verbal learning test immediate recall score; SVLT_delayed, Seoul verbal learning test delayed recall score; SVLT_rec, Seoul verbal learning test recognition score; RCFT_imm, Rey complex figure test immediate recall score; RCFT_delayed, Rey complex figure test delayed recall score; RCFT_rec, Rey complex figure test recognition score; Fluency_A, fluency score for animal; Fluency_P, fluency score for 3 Korean alphabets; Stroop_W, Stroop score for word reading; Stroop_C, Stroop score for color naming in color-word in incongruent condition; TMT, trail making test; DSF, digit span forward; DSB, digit span backward; BNT, Boston naming test (I5 items); RCFT copy, Rey complex figure test copy score.

was below -1.5 were not different between groups. These results indicate that the rMCI and sMCI groups were at the similar clinical stage of MCI.

More importantly, over a wide range of socio-demographic, clinical, and neuropsychological variables, group difference was significant only in some of neuropsychological tests. Performances in RCFT copy, Fluency_P, TMT_A, and TMT_B at baseline were significantly better in the rMCI group than those in the sMCI group. Our results are largely in line with previous longitudinal studies ${ }^{19,28,48}$ showing that the earliest signs of preclinical $\mathrm{AD}$ were captured by visuospatial and executive measures. While RCFT copy is known as a test for visuospatial ability, this test assesses the performance of reproducing a highly complex figure, which demands executive components including integrative attention and organization of 
each fraction during copying of the complex figure. ${ }^{28,49}$ However, executive function is basically an umbrella term that encompasses the set of top-down cognitive processing, such as cognitive control, decision-making, abstract thinking, planning, integrative attention, inhibition, maintenance, monitoring, set shifting, and so on. ${ }^{50}$ Not surprisingly, a single executive function test covers only part of executive components. The tests that showed a significant group difference in the current study seem to be related to cognitive control ability. Cognitive control is essentially goal-directed behavior requiring flexible allocation of mental resources via integrative attention. ${ }^{51,52}$ Cognitive control is sort of sub-concept of executive function, but greater emphasis on integrative attention and active maintenance of goals and means to achieve them. ${ }^{53,54}$ Our model for reversion prediction also suggests that individuals with MCI with higher cognitive control ability have increased probability (ie, 1.6 times higher) of reversion to $\mathrm{CN}$ compared to individuals with MCI with a lower cognitive ability. Given that phonemic fluency test and TMT are sensitive to prefrontal function, ${ }^{55-57}$ it could be speculated that better prefrontal function might be associated with the reversion from $\mathrm{MCI}$ to $\mathrm{CN}$ in the rMCI group. Future neuroimaging study could provide neural mechanism underlying the reversion from MCI to $\mathrm{CN}$. On the other hand, previous studies reported that individuals who reverted showed higher memory function at baseline than those who did not revert; $3,4,6,58$ however, we found no group difference in memory performance. The measure of memory is notably important when MCI was diagnosed. Moreover, clinical staging such as early or late MCI is often defined by episodic memory tests. ${ }^{59}$ Therefore, higher memory performance in individuals who reverted in the previous studies may indicate a cognitively earlier stage in the MCI course, consequently easier to revert. Different clinical stages could be a bias itself. In contrast, our two MCI groups were considered to be at the same cognitive stage showing similar level of memory performance and a similar number of impaired test scores.

Why do individuals with MCI revert to normal cognition? Some researchers argued that reversion could be simply false-positive errors at the initial diagnosis of MCI. ${ }^{18-20,60}$ Based on single-domain cognitive impairment or much milder cognitive impairment in reverted cases, researchers considered the impairment as normal variability and base rate of low scores in healthy elders. ${ }^{14,15,20,61}$ In the current study, however, MCI diagnosis was made by clinical interview including CDR designation (ie, evaluation cognitive change in everyday life) and consensus conference based on all available information on the subject, not solely on his or her neuropsychological performance. In addition, our rMCI group exhibited a similar level of cognitive impairment and similar distributions of the four or two clinical subtypes to the sMCI group at baseline. The frequency of MCI with only one impaired test was also not different between groups. Therefore, the possibility that MCI with milder level of cognitive impairment is related to reversion seems to be very low. That is, inclusion of false-positive diagnosis of MCI is highly unlikely in the current study.

One could also argue that practice effects at the second assessment influence reversion to $\mathrm{CN}$. As Salthouse et $\mathrm{al}^{62}$ pointed out, frequent testing could make cognitive decline obscure, especially for the elderly population. Moreover, previous large-scale longitudinal study reported that practice effect was observed even in dementia group. ${ }^{63}$ Not unexpectedly, we also observed slight improvement of visuospatial ability, memory, and executive function at the follow-up neuropsychological assessment in sMCI group as well as rMCI group. However, rANOVA revealed significant group and time interaction effects on the measures of sustained attention, memory, and fluency. The results suggested that our rMCI group showed much greater improvement in those tests than the sMCI group. One possible interpretation, although speculative, is that higher cognitive control ability in rMCI is partially attributable to such improvement. It should also be noted that we made diagnosis not solely on neuropsychological test scores but intensive diagnostic interview and CDR designation by dementia experts. For example, individuals whose neuropsychological test scores at the second evaluation were all above the $-1.5 \mathrm{SD}$ according to norms but CDR was 0.5 were not diagnosed as $\mathrm{CN}$, because their CDR was not zero. That is, classification of reversion or stable MCI groups was based on both clinical interview and neuropsychological performance. Therefore, it may seem less likely that practice effects on neuropsychological tests at the second assessment influence second diagnosis.

It is worth mentioning that we tried to include a relatively homogeneous MCI group. We excluded etiologies causing MCI that possibly exhibit cognitive fluctuations (eg, neuropsychiatric problem or vascular pathology) to examine the underlying factors for reversion from MCI to $\mathrm{CN}$ systemically. Nevertheless, a higher tendency in depression was observed in the rMCI group $(p=0.03$, but not significant at the multiple comparison corrected level). However, the depression level in general was very low 
(mean $\pm \mathrm{SD}=10.63 \pm 6.49$ for $\mathrm{rMCI}$, and $8.59 \pm 6.33$ for sMCI). Self-reported GDS is basically for screening purpose, not for diagnosis purpose. Moreover, subjects with depression were carefully examined at the stage of diagnostic interview and were excluded in the study. GDS level seems to have little impact on reversion.

Taken as a whole, our findings support that reversion from $\mathrm{MCI}$ to $\mathrm{CN}$ might not be a false-positive error but a true recovery from cognitive impairment. We postulate that individuals with MCI with higher cognitive control ability can have their cognitive function restored easier than individuals with MCI with lower cognitive control ability. Our participants all consisted of community-dwellers. Thus, they might have felt minor cognitive problems but not enough to seek medical help. However, once they knew that they had MCI, they would more take care of their cognitive health. Although the role of cognitive control in reversion from MCI to $\mathrm{CN}$ needs further validation, participants with relatively higher cognitive control ability could possibly manage to have normal cognitive function in everyday life and objective cognitive tests at a 1.5-year follow-up. Our results provide preliminary support that positive characteristics favorable to the restoration of cognitive function may be higher cognitive control ability and suggest that assessing cognitive control ability could facilitate prognosis evaluation more accurately. Furthermore, it could help to develop appropriate cognitive intervention programs in making use of cognitive control ability.

Limitations and future directions should be discussed. First, MCI was diagnosed without considering amyloid biomarkers in the current study. Inclusion of amyloid biomarker information could provide a wider window of understanding of the reversion phenomenon in the course of $\mathrm{AD}$ spectrum. Second, future multimodal neuroimaging study such as functional and structural brain imaging studies may further validate the role of cognitive control ability in the reversion from MCI to $\mathrm{CN}$. Neuroimaging information could provide neural mechanisms that might contribute to reversion from $\mathrm{MCI}$ to $\mathrm{CN}$. It could also increase predictive power for differentiation of the MCI course. Third, the current study has a relatively short follow-up period. Therefore, our results should be interpreted with caution. It is possible that some from the rMCI group could show fluctuation in cognitive function; eg, exhibiting further cognitive decline in a longer follow-up duration, as previous studies have reported. ${ }^{4,7,58}$ Therefore, a longer follow-up study would provide broader perspectives on the course of MCI. Lastly, the same neuropsychological tests used in the diagnosis were also used in the analysis. We classified reverted or stable MCI groups not solely on neuropsychological test scores but intensive diagnostic interview and CDR designation by dementia experts. Nevertheless, it may be better to use different neuropsychological test tools for diagnosis and analysis in the future study.

\section{Conclusion}

In conclusion, a considerable portion of individuals with MCI reverted to normal cognition at 1.5-year follow-up. Understanding the meaning of such reversion may have valuable implications for clinical practice and research. Our results suggest that reversion from $\mathrm{MCI}$ to $\mathrm{CN}$ might not be a false-positive error but a true recovery from cognitive impairment. Individuals with MCI with higher cognitive control ability could be restored more easily to their cognitive function than those with $\mathrm{MCI}$ with lower cognitive control ability. Therefore, assessing cognitive control ability might facilitate developing appropriate interventions for MCI as well as prognosis evaluation more accurately.

\section{Acknowledgments}

This research was supported by a Basic Science Research Program through the National Research Foundation (NRF) of Korea, funded by the Ministry of Education (NRF2017R1D1A1B03028377); the Bio \& Medical Technology Development Program of the NRF funded by the Korean government (MSIT) (No. 2016M3A9E9941914); and the Brain Research Program through the NRF funded by the Ministry of Science, ICT \& Future Planning (NRF2014M3C7A1046041).

\section{Author Contributions}

HK and EHS designed the study and EHS wrote the study protocol. JYC, HJY, and EHS wrote the draft of the manuscript and undertook the statistical analyses. JYC, HK, $\mathrm{KYC}$, JJL, KHL, and EHS collected and analyzed the data. All authors contributed toward data analysis, critically revised the paper, gave final approval of the version to be published, and agreed to be accountable for all aspects of the work.

\section{Disclosure}

The authors report no conflicts of interest in this work. 


\section{References}

1. Petersen RC, Smith GE, Waring SC, Ivnik RJ, Tangalos EG, Kokmen E. Mild cognitive impairment: clinical characterization and outcome. Arch Neurol. 1999;56(3):303-308. doi:10.1001/archneur.56.3.303

2. Lopez OL, Becker JT, Chang YF, et al. Incidence of mild cognitive impairment in the Pittsburgh Cardiovascular Health Study-Cognition Study. Neurology. 2012;79(15):1599-1606. doi:10.1212/WNL.0b01 3e31826e $25 \mathrm{fO}$

3. Pandya SY, Lacritz LH, Weiner MF, Deschner M, Woon FL. Predictors of reversion from mild cognitive impairment to normal cognition. Dement Geriatr Cogn Disord. 2017;43(3-4):204-214. doi:10.1159/000456070

4. Roberts RO, Knopman DS, Mielke MM, et al. Higher risk of progression to dementia in mild cognitive impairment cases who revert to normal. Neurology. 2014;82(4):317-325. doi:10.1212/WNL.0000000000000055

5. Tokuchi R, Hishikawa N, Kurata T, et al. Clinical and demographic predictors of mild cognitive impairment for converting to Alzheimer's disease and reverting to normal cognition. $J$ Neurol Sci. 2014;346(1-2):288-292. doi:10.1016/j.jns.2014.09.012

6. Sachdev PS, Lipnicki DM, Crawford J, et al. Factors predicting reversion from mild cognitive impairment to normal cognitive functioning: a population-based study. PLoS One. 2013;8(3):e59649. doi:10.1371/journal.pone.0059649

7. Koepsell TD, Monsell SE. Reversion from mild cognitive impairment to normal or near-normal cognition: risk factors and prognosis. Neurology. 2012;79(15):1591-1598. doi:10.1212/WNL.0b013e31826e26b7

8. Lopez OL, Jagust WJ, Dulberg C, et al. Risk factors for mild cognitive impairment in the Cardiovascular Health Study Cognition Study: part 2. Arch Neurol. 2003;60(10):1394-1399. doi:10.1001/archneur.60.10.1394

9. Ravaglia G, Forti P, Montesi F, et al. Mild cognitive impairment: epidemiology and dementia risk in an elderly Italian population. $J$ Am Geriatr Soc. 2008;56(1):51-58. doi:10.1111/j.1532-5415.2007.01503.x

10. Clark LR, Delano-Wood L, Libon DJ, et al. Are empirically-derived subtypes of mild cognitive impairment consistent with conventional subtypes? J Int Neuropsychol Soc. 2013;19(6):635-645. doi:10.1017/ S1355617713000313

11. Nettiksimmons J, DeCarli C, Landau S, Beckett L. Alzheimer's disease neuroimaging I. Biological heterogeneity in ADNI amnestic mild cognitive impairment. Alzheimers Dement. 2014;10(5):511-521 e511. doi:10.1016/j.jalz.2013.09.003

12. Flicker C, Ferris SH, Reisberg B. Mild cognitive impairment in the elderly: predictors of dementia. Neurology. 1991;41(7):1006-1009. doi: $10.1212 / w n 1.41 .7 .1006$

13. Larrieu S, Letenneur L, Orgogozo JM, et al. Incidence and outcome of mild cognitive impairment in a population-based prospective cohort. Neurology. 2002;59(10):1594-1599. doi:10.1212/01. wnl.0000034176.07159.f8

14. Brooks BL, Iverson GL, White T. Substantial risk of "Accidental MCI" in healthy older adults: base rates of low memory scores in neuropsychological assessment. J Int Neuropsychol Soc. 2007;13 (3):490-500. doi:10.1017/S1355617707070531

15. de Rotrou J, Wenisch E, Chausson C, Dray F, Faucounau V, Rigaud AS. Accidental MCI in healthy subjects: a prospective longitudinal study. Eur J Neurol. 2005;12(11):879-885. doi:10.1111/j.1468-1331.2005.01100.x

16. Gao Q, Gwee X, Feng L, et al. Mild cognitive impairment reversion and progression: rates and predictors in community-living older persons in the singapore longitudinal ageing studies cohort. Dement Geriatr Cogn Dis Extra. 2018;8(2):226-237. doi:10.1159/000488936

17. Aretouli E, Tsilidis KK, Brandt J. Four-year outcome of mild cognitive impairment: the contribution of executive dysfunction. Neuropsychology. 2013;27(1):95-106. doi:10.1037/a0030481

18. Brooks BL, Iverson GL, Holdnack JA, Feldman HH. Potential for misclassification of mild cognitive impairment: a study of memory scores on the Wechsler Memory Scale-III in healthy older adults. J Int Neuropsychol Soc. 2008;14(3):463-478. doi:10.1017/S1355617708080521
19. Edmonds EC, Delano-Wood L, Clark LR, et al. Susceptibility of the conventional criteria for mild cognitive impairment to false-positive diagnostic errors. Alzheimers Dement. 2015;11(4):415-424. doi:10.1016/j.jalz.2014.03.005

20. Oltra-Cucarella J, Sanchez-Sansegundo M, Lipnicki DM, et al. Using base rate of low scores to identify progression from amnestic mild cognitive impairment to Alzheimer's disease. J Am Geriatr Soc. 2018;66(7):1360-1366. doi:10.1111/jgs.15412

21. Sugarman MA, Alosco ML, Tripodis Y, Steinberg EG, Stern RA. Neuropsychiatric symptoms and the diagnostic stability of mild cognitive impairment. J Alzheimers Dis. 2018;62(4):1841-1855. doi: 10.3233/JAD-170527

22. Teng E, Tingus KD, Lu PH, Cummings JL. Persistence of neuropsychological testing deficits in mild cognitive impairment. Dement Geriatr Cogn Disord. 2009;28(2):168-178. doi:10.1159/000235732

23. Cooper DB, Lacritz LH, Weiner MF, Rosenberg RN, Cullum CM. Category fluency in mild cognitive impairment: reduced effect of practice in test-retest conditions. Alzheimer Dis Assoc Disord. 2004;18(3):120-122.

24. Duff K, Beglinger LJ, Van Der Heiden S, et al. Short-term practice effects in amnestic mild cognitive impairment: implications for diagnosis and treatment. Int Psychogeriatr. 2008;20(5):986-999. doi:10.1017/S1041610208007254

25. Duff K, Lyketsos CG, Beglinger LJ, et al. Practice effects predict cognitive outcome in amnestic mild cognitive impairment. Am J Geriatr Psychiatry. 2011;19(11):932-939. doi:10.1097/JGP.0b013e318209dd3a

26. Dodge HH, Wang CN, Chang CC, Ganguli M. Terminal decline and practice effects in older adults without dementia: the MoVIES project. Neurology. 2011;77(8):722-730. doi:10.1212/WNL.0b013e31822b0068

27. Duff K, Beglinger LJ, Moser DJ, Paulsen JS, Schultz SK, Arndt S. Predicting cognitive change in older adults: the relative contribution of practice effects. Arch Clin Neuropsychol. 2010;25(2):81-88. doi:10.1093/arclin/acp105

28. Seo EH, Kim H, Choi KY, Lee KH, Choo IH. Pre-mild cognitive impairment: can visual memory predict who rapidly convert to mild cognitive impairment? Psychiatry Investig. 2018;15(9):869-875. doi:10.30773/pi.2018.07.29.1

29. Seo EH, Kim H, Lee KH, Choo IH. Altered executive function in pre-mild cognitive impairment. J Alzheimers Dis. 2016;54(3):933940. doi:10.3233/JAD-160052

30. Winblad B, Palmer K, Kivipelto M, et al. Mild cognitive impairmentbeyond controversies, towards a consensus: report of the International Working Group on Mild Cognitive Impairment. J Intern Med. 2004;256 (3):240-246. doi:10.1111/j.1365-2796.2004.01380.x

31. Fazekas F, Chawluk JB, Alavi A, Hurtig HI, Zimmerman RA. MR signal abnormalities at $1.5 \mathrm{~T}$ in Alzheimer's dementia and normal aging. AJR Am J Roentgenol. 1987;149(2):351-356. doi:10.2214/ ajr.149.2.351

32. Hughes CP, Berg L, Danziger WL, Coben LA, Martin RL. A new clinical scale for the staging of dementia. $\mathrm{Br} J$ Psychiatry. 1982;140:566-572. doi:10.1192/bjp.140.6.566

33. Morris JC. The Clinical Dementia Rating (CDR): current version and scoring rules. Neurology. 1993;43(11):2412-2414. doi:10.1212/ wn1.43.11.2412-a

34. Kang SJ, Choi SH, Lee BH, Kwon JC, Na DL, Han SH. Validity and reliability of Korean-Instrumental activities of daily living. Korean $J$ Neurosci. 2002;20:8-14.

35. Organization WH. The Asia-Pacific Perspective: Redefining Obesity and Its Treatment. Geneva: World Health Organization Western Pacific Regional Office; 2000.

36. Yang DW, Cho BL, Chey JY, Kim SY, Kim BS. The development and validation of Korean Dementia Screening Questionnaire (KDSQ). J Korean Neurol Assoc. 2002;20(2):135-141.

37. Youn JC, Kim KW, Lee DY, et al. Development of the Subjective memory complaints questionnaire. Dement Geriatr Cogn Disord. 2009;27(4):310-317. doi:10.1159/000205512 
38. Kim JY, Park JH, Lee JJ, et al. Standardization of the korean version of the geriatric depression scale: reliability, validity, and factor structure. Psychiatry Investig. 2008;5(4):232-238. doi:10.4306/pi.2008.5.4.232

39. Kang Y, Jang S, Na DL. Seoul Neuropsychological Screenign Battery (SNSB). 2nd ed. Seoul: Human Brain Research \& Consulting Co; 2012.

40. Nijenhuis JT, Choi KY, Choi YY, et al. Differences between APOE carriers and non-APOE carriers on neurocognitive tests: Jensen effects? Am J Alzheimers Dis Other Demen. 2018;33(6):353-361. doi: $10.1177 / 1533317518765895$

41. American Psychiatric Association. Diagnostic and Statistical Manual of Mental Disorders. 4th ed. Washington, DC: American Psychiatric Association Press; 1994.

42. McKhann G, Drachman D, Folstein M, Katzman R, Price D, Stadlan EM. Clinical diagnosis of Alzheimer's disease: report of the NINCDS-ADRDA Work Group under the auspices of department of health and human services task force on Alzheimer's disease. Neurology. 1984;34(7):939-944. doi:10.1212/wnl.34.7.939

43. Benjamini Y, Hochberg Y. Controlling the false discovery rate: a practical and powerful approach to multiple testing. J $R$ Stat Soc Ser B. 1995;57(1):289-300. doi:10.1111/rssb.1995.57.issue-1

44. Manly JJ, Tang MX, Schupf N, Stern Y, Vonsattel JP, Mayeux R. Frequency and course of mild cognitive impairment in a multiethnic community. Ann Neurol. 2008;63(4):494-506. doi:10.1002/ ana. 21326

45. Malek-Ahmadi M. Reversion from mild cognitive impairment to normal cognition: a meta-analysis. Alzheimer Dis Assoc Disord. 2016;30(4):324-330. doi:10.1097/WAD.0000000000000145

46. Petersen RC. Does the source of subjects matter?: absolutely! Neurology. 2010;74(22):1754-1755. doi:10.1212/WNL.0b013e3181e533e7

47. Roh HW, Hong CH, Lee Y, et al. Clinical conversion or reversion of mild cognitive impairment in community versus hospital based studies: GDEMCIS (Gwangju Dementia and Mild Cognitive Impairment Study) and CREDOS (Clinical Research Center for Dementia of South Korea). J Alzheimers Dis. 2016;53(2):463-473. doi:10.3233/JAD-160341

48. Johnson DK, Storandt M, Morris JC, Galvin JE. Longitudinal study of the transition from healthy aging to Alzheimer disease. Arch Neurol. 2009;66(10):1254-1259. doi:10.1001/archneurol.2009.158

49. Watanabe K, Ogino T, Nakano K, et al. The Rey-Osterrieth complex figure as a measure of executive function in childhood. Brain Dev. 2005;27(8):564-569. doi:10.1016/j.braindev.2005.02.007

50. Diamond A. Executive functions. Annu Rev Psychol. 2013;64:135168. doi:10.1146/annurev-psych-113011-143750
51. Badre D. Cognitive control, hierarchy, and the rostro-caudal organization of the frontal lobes. Trends Cogn Sci. 2008;12(5):193-200. doi:10.1016/j.tics.2008.02.004

52. Mackie MA, Van Dam NT, Fan J. Cognitive control and attentional functions. Brain Cogn. 2013;82(3):301-312. doi:10.1016/j.bandc. 2013.05.004

53. Nigg JT. Annual research review: on the relations among self-regulation, self-control, executive functioning, effortful control, cognitive control, impulsivity, risk-taking, and inhibition for developmental psychopathology. J Child Psychol Psychiatry. 2017;58(4):361-383. doi:10.1111/jcpp. 12675

54. Miller EK, Cohen JD. An integrative theory of prefrontal cortex function. Annu Rev Neurosci. 2001;24:167-202. doi:10.1146/annurev.neuro. 24.1.167

55. Henry JD, Crawford JR. A meta-analytic review of verbal fluency performance following focal cortical lesions. Neuropsychology. 2004;18(2):284-295. doi:10.1037/0894-4105.18.2.284

56. Meinzer M, Flaisch T, Wilser L, et al. Neural signatures of semantic and phonemic fluency in young and old adults. J Cogn Neurosci. 2009;21(10):2007-2018. doi:10.1162/jocn.2009.21219

57. Varjacic A, Mantini D, Demeyere N, Gillebert CR. Neural signatures of Trail Making Test performance: evidence from lesion-mapping and neuroimaging studies. Neuropsychologia. 2018;115:78-87. doi:10.1016/j. neuropsychologia.2018.03.031

58. Kaduszkiewicz H, Eisele M, Wiese B, et al. Prognosis of mild cognitive impairment in general practice: results of the German AgeCoDe study. Ann Fam Med. 2014;12(2):158-165. doi:10.1370/afm.1596

59. Aisen PS, Petersen RC, Donohue M, Weiner MW. Alzheimer's disease neuroimaging I. Alzheimer's disease neuroimaging initiative 2 clinical core: progress and plans. Alzheimers Dement. 2015;11 (7):734-739. doi:10.1016/j.jalz.2015.05.005

60. Park MH, Han C. Is there an MCI reversion to cognitively normal? Analysis of Alzheimer's disease biomarkers profiles. Int Psychogeriatr. 2015;27(3):429-437. doi:10.1017/S1041610214002129

61. Palmer BW, Boone KB, Lesser IM, Wohl MA. Base rates of "impaired" neuropsychological test performance among healthy older adults. Arch Clin Neuropsychol. 1998;13(6):503-511.

62. Salthouse TA. Frequent assessments may obscure cognitive decline. Psychol Assess. 2014;26(4):1063-1069. doi:10.1037/pas0000007

63. Machulda MM, Pankratz VS, Christianson TJ, et al. Practice effects and longitudinal cognitive change in normal aging vs. incident mild cognitive impairment and dementia in the Mayo Clinic Study of Aging. Clin Neuropsychol. 2013;27(8):1247-1264. doi:10.1080/ 13854046.2013 .836567
Neuropsychiatric Disease and Treatment

\section{Publish your work in this journal}

Neuropsychiatric Disease and Treatment is an international, peerreviewed journal of clinical therapeutics and pharmacology focusing on concise rapid reporting of clinical or pre-clinical studies on a range of neuropsychiatric and neurological disorders. This journal is indexed on PubMed Central, the 'PsycINFO' database and CAS, and is the official journal of The International Neuropsychiatric Association (INA). The manuscript management system is completely online and includes a very quick and fair peer-review system, which is all easy to use. Visit http://www.dovepress.com/testimonials.php to read real quotes from published authors. 